Western University

Scholarship@Western

Brain and Mind Institute Researchers'

Publications

Brain and Mind Institute

$1-1-2021$

\title{
The relationship between cognitive ability and BOLD activation across sleep-wake states
}

\author{
Zhuo Fang \\ Université d'Ottawa, Faculté de Médecine \\ Dylan M. Smith \\ Université d'Ottawa, Faculté de Médecine \\ Evan Houldin \\ University of Ottawa \\ Laura Ray \\ The University of Western Ontario \\ Adrian M. Owen \\ The University of Western Ontario, uwocerc@uwo.ca
}

See next page for additional authors

Follow this and additional works at: https://ir.lib.uwo.ca/brainpub

Citation of this paper:

Fang, Zhuo; Smith, Dylan M.; Houldin, Evan; Ray, Laura; Owen, Adrian M.; and Fogel, Stuart, "The relationship between cognitive ability and BOLD activation across sleep-wake states" (2021). Brain and Mind Institute Researchers' Publications. 947.

https://ir.lib.uwo.ca/brainpub/947 


\section{Authors}

Zhuo Fang, Dylan M. Smith, Evan Houldin, Laura Ray, Adrian M. Owen, and Stuart Fogel 


\title{
The relationship between cognitive ability and BOLD activation across sleep-wake states
}

\author{
Zhuo Fang ${ }^{1} \cdot$ Dylan M. Smith ${ }^{1,2} \cdot$ Evan Houldin $^{2} \cdot$ Laura Ray $^{3} \cdot$ Adrian M. Owen $^{3} \cdot$ Stuart Fogel $^{1,2,4}$
}

Accepted: 7 July 2021

(c) The Author(s), under exclusive licence to Springer Science+Business Media, LLC, part of Springer Nature 2021

\begin{abstract}
The sleep spindle, a waxing and waning oscillation in the sigma frequency range, has been shown to correlate with fluid intelligence; i.e. the ability to use logic, learn novel rules/patterns, and solve problems. Using simultaneous EEG and fMRI, we previously identified the neural correlates of this relationship, including activation of the thalamus, bilateral putamen, medial frontal gyrus, middle cingulate cortex, and precuneus. However, research to date has focussed primarily on non-rapid eye movement (NREM) sleep, and spindles per se, thus overlooking the possibility that brain activity that occurs in other sleep-wake states might also be related to cognitive abilities. In our current study, we sought to investigate whether brain activity across sleep/wake states is also related to human intelligence in $\mathrm{N}=29$ participants. During NREM sleep, positive correlations were observed between fluid intelligence and blood oxygen level dependent (BOLD) activations in the bilateral putamen and the paracentral lobule/precuneus, as well as between short-term memory (STM) abilities and activity in the medial frontal cortex and inferior frontal gyrus. During wake, activity in bilateral postcentral gyri and occipital lobe was positively correlated with short-term memory abilities. In participants who experienced REM sleep in the scanner, fluid intelligence was positively associated with midbrain activation, and verbal intelligence was associated with right postcentral gyrus activation. These findings provide evidence that the relationship between sleep and intellectual abilities exists beyond sleep spindles.
\end{abstract}

Keywords Sleep $\cdot$ Intelligence $\cdot$ Reasoning abilities $\cdot$ Verbal abilities $\cdot$ Short-term memory $\cdot$ NREM $\cdot$ REM $\cdot$ fMRI $\cdot$ EEG

\section{Introduction}

While a relationship between sleep and human health has long been established (Belloc \& Breslow, 1972; Worley, 2018), the link between sleep and human intelligence is less clear. General human intelligence can be described as an emergent construct arising from multiple specialized cognitive components (e.g., related to reasoning and verbal

Stuart Fogel

sfogel@uottawa.ca

http://socialsciences.uottawa.ca/sleep-lab/

1 University of Ottawa Brain \& Mind Research Institute, University of Ottawa, Ottawa, Canada

2 Sleep Unit, University of Ottawa Institute of Mental Health Research at The Royal, University of Ottawa, Ottawa, Canada

3 The Brain and Mind Institute, Western University, London, Canada

4 School of Psychology, University of Ottawa, Ottawa, ON K1N 6N5, Canada abilities, and the capacity to maintain information in shortterm memory). These specialized abilities are often highly correlated; however, they are also associated with dissociable and distinct patterns of neural activation (Hampshire et al., 2012), which are thought to reflect the neural substrates which support these unique cognitive abilities. While there is much evidence that sleep is integral for optimizing cognitive processes (e.g., neuronal replay, memory reactivation, strengthening, transformation and optimization) across multiple domains (Diekelmann, 2014), it remains unclear whether an individual's unique cognitive strengths and weaknesses are related to, or perhaps even supported by brain activity across different sleep stages.

Recently, a strong association was found between sleep duration and performance on cognitive tasks; individuals who achieve an optimal amount of sleep (7-9 h) tend to exhibit better cognition (Wild et al., 2018). Similarly, sleep deprivation has been associated with deficits in vigilance, attention, and working memory, as well as attenuated reward and emotional processing (Krause et al., 2017; Lim 
\& Dinges, 2010). Associations between optimal health, sleep and cognition have been observed across the lifespan, and this relationship may be particularly important during development, as well as in old age (Dzierzewski et al., 2018; Tarokh et al., 2016), with effect sizes being consistent yet small across studies (Astill et al., 2012; Lo et al., 2016). Importantly, such effects are also sensitive to variations in measurement (e.g., self-report vs. actigraphy vs. polysomnography), quantification of sleep (e.g., sleep efficiency vs. sleep duration), and other factors (Ujma et al., 2020) which make direct comparisons between studies difficult. A large number of studies have specifically investigated the relationship between sleep and memory consolidation, and it has been proposed that sleep may also contribute to the integration of new memories into existing knowledge, optimizing neural networks for storage and retrieval of biologically important information, as well as facilitating associations between distinct memories and enabling more novel, creative thought (Walker, 2009). Thus, it seems that sleep's role in the maintenance of neural network homeostasis may have implications for a wide breadth of cognitive functions.

Many modern electroencephalography (EEG) studies have specifically investigated the relationship between general mental ability and the presence of waxing and waning neural oscillations known as sleep spindles. Much like the above-described link between cognition and macrostructural measures of sleep, the relationship between cognition and sleep spindles is consistent yet small (Bódizs et al., 2005; Pesonen, et al., 2019; Schabus et al., 2006; Ujma et al., 2014; Ujma, 2018), with spindle amplitude showing the most promise as a biomarker for human intelligence. However, comparisons between studies are confounded by small sample sizes, variations in detection algorithms, and publication bias (Ujma et al., 2020). Other studies have found trait-like relationships between spindles and visuo-spatial memory (Bódizs et al., 2008), non-verbal fluid intelligence (Fang et al., 2017; Fogel et al., 2007; Reynolds et al., 2018; Ujma et al., 2015), as well as individual differences in performance on a word-pair task (Schabus et al., 2004). These studies seem to suggest that the sleep spindle may be an indicator of the health or efficiency of the neural circuitry supporting intelligence, and this association is most commonly found when assessing fluid intelligence specifically. However, the above-mentioned confounding variables underline the need for replication of these individual findings, controlling for factors which are known correlates of intelligence, such as age and brain development. Furthermore, it is not yet clear if intelligence is uniquely linked to spindles over and above other yet to-be-identified and potentially confounding or mediating aspects of sleep and their functions, although a recent study by our group suggests that a few of the likely candidate factors such as sleep quality and chronotype do not explain the relationship between spindles and fluid intelligence, specifically (Fang et al., 2017).

Previously, using simulations based on neuroimaging data, we demonstrated that different components of intelligence are reflected in distinct brain networks (Hampshire et al., 2012). Specifically, comparing factor models of brain activity in overlapping functional networks with individual differences in performance on twelve cognitive tasks, 3 distinct orthogonal factors were found to account for an individuals' general cognitive ability; the (1) Reasoning factor, which involves fluid intelligence; e.g., problem solving, logic, complex pattern recognition, (2) the Short Term Memory factor, involving e.g., maintenance and recall of newly acquired information, including visuospatial working memory, and finally, (3) the Verbal factor, representing e.g., language-dependent processing. Using simultaneous EEG-fMRI sleep recordings, we found that brain activation time-locked to spindle events correlated with the Reasoning factor, but not the Short-Term Memory factor, nor the Verbal Factor (Fang et al., 2019b). Furthermore, a subsequent study employing psychophysiological interaction analysis of EEG-fMRI recordings found that the extent of the functional connectivity of the cortical-striatal circuitry and the thalamo-cortical circuitry was specifically related to the Reasoning factor, but not the Short-Term Memory nor Verbal factors (Fang et al., 2019a). These findings complemented previous work which found a unique relationship between spindle amplitude and the Reasoning factor over-and-above Short-Term Memory and Verbal factors (Fang et al., 2017).

While these studies support the hypothesized link specifically between sleep spindle-dependent brain activation and fluid intelligence, the question remains whether other, non-spindle-related brain activity during sleep might also be associated with intelligence across sleep-wake states, perhaps in cognitive domains other than reasoning abilities, such as verbal or short-term memory abilities. According to previous studies, both REM and slow wave sleep duration have been positively associated with performance on executive function tasks, and slow-wave sleep has been positively associated with processing speed over a number of cognitive domains (Della Monica et al., 2018; Smith et al., 2004), suggesting that non-rapid eye movement stage 2 (NREM2) sleep, where spindles predominate, is not unique in its association with intelligence. Similarly, in a large $(\mathrm{N}=151)$ study (Ujma et al., 2017), the EEG power spectrum of healthy individuals during sleep was compared to performance on nonverbal intelligence tests, and multiple sexually dimorphic effects were observed, with the most consistent effects found in REM sleep, where females uniquely exhibited a positive linear relationship between REM anterior beta power and cognition. Taken together, these studies provide converging evidence that sleep-dependent neural activity apart from spindles is also associated with intelligence. Thus it may be 
possible that spindle-dependent activity represents only one part of a more complex relationship between brain activity and intelligence during sleep.

In order to examine this possibility, we further analyzed our dataset from (Fang et al., 2019a, 2019b), using sleep stages in lieu of spindles as events-of-interest, to elucidate whether neural activation within different stages of sleep (regardless of spindle-presence) were associated with human intellectual abilities. Specifically, the extent of brain activations related to Reasoning, Short-Term Memory, and Verbal factors were investigated during NREM, REM, and during wake. We hypothesized that the extent of activation in the striatal-cortical network during NREM2 sleep would be correlated with Reasoning, but not Verbal factors. Conversely, brain activation during REM sleep would be correlated with Verbal, but not Reasoning abilities. Neither Reasoning nor Verbal factors were expected to be correlated with the extent of wake BOLD activations; although there is evidence that resting state functional connectivity during wake has been associated with fluid intelligence (Santarnecchi et al., 2017), it was not expected that the activation of individual brain regions would show a correlation with Reasoning or Verbal factors. Short-term memory has similarly been associated with resting state functional connectivity (Stevens et al., 2012), however, due to a lack of studies exploring STM and sleep-wake states, we did not expect any associations between STM factor and BOLD activation in NREM, REM, or Wake.

\section{Methods \& Materials}

\section{Participants}

A total of 35 healthy right-handed adults (20 females) between 20-35 years old $(\mathrm{M}=23.69, \mathrm{SD}=3.57)$ were recruited to participate in this study. Participants were screened to ensure healthy lifestyles, no shift work, no medications known to interfere with sleep, no history of sleep disorders, neurological, psychiatric, or signs of mental health issues (see Fang et al., 2019a for detailed inclusion/ exclusion criteria). Participants were provided with informed written consent before participation. All study procedures and methods adhered to the Declaration of Helsinki and were approved by the Western University Health Science research ethics board.

At least five minutes of uninterrupted NREM sleep was required for data analysis purposes. Among the 35 participants recruited in the study, only 5 participants did not meet this criteria, and one participant did not complete the Cambridge Brain Sciences online test. The remaining 29 participants $(\mathrm{M}=23.97, \mathrm{SD}=3.83,17$ female $)$ were included in the analyses, and 8 of them reached REM sleep (mean total sleep time $=44.20 \pm 23.84 \mathrm{~min}$; sleep latency $=8.16 \pm 10.11 \mathrm{~min}$; sleep onset time $=22 \mathrm{~h} 22 \pm 25 \mathrm{~min})$. All $(\mathrm{N}=29)$ participants had at least $14.67 \mathrm{~min}$ of sleep during the EEG-fMRI sleep recording. The detailed sleep architecture parameters are reported in Table 1 and (Fang, et al., 2019b).

\section{Experimental procedure}

Following the initial screening session, eligible participants were given a guide to complete the online Cambridge Brain Sciences (CBS) trials, a sleep diary and an actiwatch to verify compliance with instructions to maintain a normal sleep-wake cycle (bedtime between 10 pm-12am, waketime between 7am-9am). During the EEG-fMRI sleep recording night, the scanning session was conducted between $21 \mathrm{~h} 00$ and $24 \mathrm{~h} 00$, at the end of which simultaneous EEG-fMRI was recorded while participants slept in the scanner. The scan started at $21 \mathrm{~h}$, at which point, the EEG equipment was installed and tested. This was followed by localizer scans, a T1 MPRAGE structural scan, and an awake resting state scan. These procedures took up to an hour to complete. The sleep recording ('lights out') started around $22 \mathrm{~h} 00$, within the range of the participants' habitual bedtime. Following the sleep session, participants were allowed to sleep in the nearby sleep laboratory for the remainder of the night.

\section{Tests of cognitive abilities}

The Cambridge Brain Sciences (CBS) battery was used to assess participants' cognitive abilities (Hampshire et al., 2012; Wild et al., 2018). CBS is a web-based battery of 12 cognitive tests that measures a broad range of cognitive abilities including reasoning, problem solving, planning, attention, and memory (https://www.cambridgebrainscienc es.com/tests). A recent study, based on scores from a population-sized pool of 44,600 participants (Hampshire et al.,

Table 1 Sleep architecture from EEG-fMRI recording sessions

\begin{tabular}{llr}
\hline & M & SD \\
\hline & Sleep Architecture & \\
Wake $(\min )(\mathrm{N}=26)$ & 26.87 & 20.25 \\
NREM1 (min) $(\mathrm{N}=26)$ & 5.84 & 4.38 \\
NREM2 (min) $(\mathrm{N}=29)$ & 23.87 & 14.50 \\
SWS (min) $(\mathrm{N}=20)$ & 14.77 & 17.17 \\
NREM (min) & 39.29 & 19.33 \\
REM (N=8) & 17.80 & 10.76 \\
Total Sleep & 44.20 & 23.84 \\
Sleep latency & 8.16 & 10.11 \\
\hline
\end{tabular}

Abbreviations: non-rapid eye movement sleep (NREM); stage 1 sleep (NREM1); stage 2 sleep (NREM2); slow wave sleep (SWS); rapid eye movement sleep (REM) 
2012), revealed three factors that govern performance across the Cambridge Brain Sciences tests. These factors have been described as "Reasoning" (i.e., fluid intelligence), "ShortTerm Memory" (STM) and "Verbal" abilities (i.e., crystalized intelligence). For clarity, this paper will use capitalization in order to distinguish the factors "Reasoning" "Verbal" and "STM" from the general terms reasoning, verbal, and short-term memory. The Reasoning factor is best described in terms of performance on five tests adapted from the cognitive literature, including deductive reasoning (Cattell, 1940), spatial rotation (Silverman et al., 2000), feature match (Treisman \& Gelade, 1980), spatial planning (Shallice, 1982), and interlocking polygons (Folstein et al., 1975). STM is best described in terms of four tests, including visuospatial working memory (Inoue et al., 2000), spatial span (Corsi, 1972), paired associates (Collins et al., 1998; Gould et al., 2006), and self-ordered search (Collins et al., 1998). Finally, Verbal ability is best captured by performance on three tests, including verbal reasoning (Baddeley, 1968), color-word remapping (Stroop, 1935), and digit span (Wecshler, 1981). Importantly, the Reasoning, STM, and Verbal factors are not simply aggregates of scores on domain-specific tasks. Rather, they are statistically orthogonal factors, and represent unique abilities which differentially influence performance on all tasks, with distinct functional neural correlates (Hampshire et al., 2012). The raw scores from each of the 12 tests were normalized using the mean and standard deviation obtained from a large, young population $(\mathrm{N}=44,600$; age 20 to 35) of participants who completed the CBS test battery (Hampshire et al., 2012). Each test score was then weighted using varimax-rotated orthogonal factor loadings from Hampshire et al., (2012) in order to create the Reasoning, Short-Term Memory and Verbal factors. Finally, these factors were transformed to standard scores by mean centering to 100 with a standard deviation of 15 , so that test scores were readily comparable to results from similar studies that employed test batteries tapping into Reasoning and Verbal abilities, such as the Multidimensional Aptitude Battery - II (Fogel et al., 2007; Fogel \& Smith, 2006) and other commonly used batteries of cognitive abilities (e.g., Wechsler Adult Intelligence Scale). The descriptive statistics of each factor are shown in Table 2.

\section{Polysomnographic recording and analysis}

EEG was recorded using a 64-channel magnetic resonance (MR)-compatible EEG cap which included one electrocardiogram (ECG) lead (Braincap MR, Easycap, Herrsching, Germany) and recorded via two MR-compatible 32-channel amplifiers (Brainamp MR plus, Brain Products $\mathrm{GmbH}$, Gilching, Germany). EEG hardware specifications, recording parameters and procedures are described in detail in (Fang, et al., 2019b).
Table 2 Descriptive statistics of the 3 Cambridge Brain Sciences factors (Reasoning, Short-term memory (STM) and Verbal)

\begin{tabular}{lllr}
\hline IQ Measures & Range & Mean (SD) & Median \\
\hline Reasoning & $78.84-108.17$ & $95.65(7.20)$ & 96.46 \\
STM & $84.38-115.33$ & $101.60(6.77)$ & 102.30 \\
Verbal & $88.51-110.92$ & $99.62(5.12)$ & 99.52 \\
\hline
\end{tabular}

EEG scanner artifacts were removed in two separate steps: (1) MRI gradient artifacts were removed using an adaptive average template subtraction method (Allen et al., 2000) implemented in Brain Products Analyzer, and downsampled to $250 \mathrm{~Hz}$, and, (2) the r-peaks in the ECG were semi-automatically detected, each detection visually verified and manually corrected to be precisely aligned with the maximum of the r-peak. Then, adaptive template subtraction (Allen et al., 1998) was used to remove ballistocardiographic (BCG) artifacts time-locked to the R-peak of the cardiac rhythm QRS complex. Finally, EEG was low-pass filtered $(60 \mathrm{~Hz})$ and re-referenced to the averaged mastoids. After these steps, the resulting quality of the data was visually verified and we inspected the amplitude of the residual artifacts time-locked to the r-peaks. Any remaining BCG residual artifact was removed using independent component analysis (ICA) based approach (Mantini et al., 2007; Srivastava et al., 2005) if the peak of the maximum amplitude of the residual artifact exceeded $3 \mu \mathrm{V}$ during the $\mathrm{QRS}$ complex (e.g., 0 to $600 \mathrm{~ms}$ ).

Following the artifact correction, sleep stages were scored in accordance with standard criteria (Iber, et al., 2007) using the "VisEd Marks" toolbox (https://github.com/jadesjardi ns/vised_marks) for EEGlab (Delorme \& Makeig, 2004). Twenty-second long EEG epochs were classified into the different sleep stages [i.e., wake, NREM1, NREM2, slow wave sleep (SWS) and REM]. In the current study, there was insufficient NREM1 and SWS across participants, and so we focus on the brain activations during wake, NREM, and REM sleep. For the purpose of conducting the fMRI general linear model (GLM) analyses, we extracted every continuous 20-s epoch (9-10 image volumes) for each sleep-wake stage as an event of interest. There were at least 15 events ( 138 image volumes) for each stage used for the GLM. The detailed information of the selected events during each sleep stage is shown in Supplementary Table S1.

\section{MRI imaging acquisition and analysis}

Recording Parameters. fMRI was performed on a 3.0 T Magnetom Prisma magnetic resonance imaging system (Siemens, Erlangen, Germany) using a 64-channel head coil. High-resolution anatomic images were acquired using a standard 3D Multislice MPRAGE sequence $(\mathrm{TR}=2300 \mathrm{~ms}, \mathrm{TE}=2.98 \mathrm{~ms}$, 
$\mathrm{TI}=900 \mathrm{~ms}, \mathrm{FA}=9^{\circ}$, 176 slices, $\mathrm{FoV}=256 \times 256 \mathrm{~mm}^{2}$, matrix size $=256 \times 256 \times 176$, voxel size $\left.=1 \times 1 \times 1 \mathrm{~mm}^{3}\right)$. During the sleep session, T2*-weighted fMRI images were acquired with a gradient echo-planar imaging (EPI) sequence using axial slice orientation $\left(\mathrm{TR}=2160 \mathrm{~ms}, \mathrm{TE}=30 \mathrm{~ms}, \mathrm{FA}=90^{\circ}\right.$, 40 transverse slices, $3 \mathrm{~mm}$ slice thickness, $10 \%$ inter-slice gap, $\mathrm{FoV}=220 \times 220 \mathrm{~mm}^{2}$, matrix size $=64 \times 64 \times 40$, voxel size $=3.44 \times 3.44 \times 3 \mathrm{~mm}^{3}$ ). Among all participants, up to $2.25 \mathrm{~h}$ of sleep EEG-fMRI data was acquired.

Image Preprocessing. Functional images were preprocessed and analyzed using SPM8 (http://www.fil.ion.ucl.ac. uk/spm/software/spm8/; Welcome Department of Imaging Neuroscience, London, UK) implemented in MATLAB (ver. 8.5 R2015a) for Windows (Microsoft, Inc. Redmond, WA). For each participant, functional images were corrected for slice acquisition time differences and realigned to correct head motion using rigid body transformation. A mean realigned image was then created from the resulting images. The structural T1-image was coregistered to this mean volume of functional images. Using DARTEL in SPM8, the coregistered structural images were segmented into grey matter, white matter and cerebrospinal fluid, and an average subject-based template was created. All functional and anatomical images were then spatially normalized using the resulting template, which was generated from the structural scans. Finally, spatial smoothing was applied on all functional images (Gaussian kernel, $8 \mathrm{~mm}$ full-width at halfmaximum (FWHM).

\section{Sleep-wake stage-related first-level general linear model}

Among all participants, the first-level GLM was fit when considering brain responses during each 20-s sleep stage epoch (wake, NREM, REM), the onsets and durations of all events in each sleep stage were convolved with the standard canonical hemodynamic response function (HRF). Nuisance variables in the model included: the Friston-24 head motion parameters (Friston et al., 1996), as well as, the mean white matter intensity and the mean cerebrospinal fluid intensity for each participant. Slow drifts were removed from the time series using a high pass filter with a cut-off period of $128 \mathrm{~s}$. Serial correlations in the fMRI signal were estimated using an autoregressive (order 1) plus white noise model and a restricted maximum likelihood (ReML) algorithm. These analyses generated contrast maps of the t-statistic [SPM(t)] for wake, NREM, and REM respectively.

\section{Correlation between CBS scores and brain activations modulated by sleep-wake states}

To investigate the relationship between the magnitude of the brain activation during different sleep stages and the cognitive abilities assessed by the CBS factors, whole-brain spatial multiple regression analyses were conducted at the group-level using the SPM8 toolbox. Sleep stage-related contrast maps of the t-statistic [SPM(t)] from 1st-level GLM were selected, and cognitive test scores for each factor (i.e., Reasoning, Verbal, and Short-Term Memory) were entered as covariates of interest in the described GLMs together. Gender and whole brain volume were included in the models as variables of non-interest. Multiple regression analyses were conducted at the whole-brain level to identify which brain regions recruited during each sleep stage were correlated to each CBS factor. Statistical inferences were performed at a threshold of uncorrected $p<0.005$ at the wholebrain level and $\mathrm{p}<0.05$, family wise error (FWE) corrected or small volume corrected (SVC) at the cluster level. In order to assess the specificity of the results of our cognitive domain analyses, this same analysis was also performed using a general ability score common to all 12 tasks in lieu of the three domain factors, by extracting the first order factor from a principal components analysis performed in SPSS version 26 (IBM Corp., 2019), termed the "g-factor".

\section{Results}

\section{Correlation between Reasoning Abilities and brain activations modulated by sleep-wake state}

As shown in Fig. 1A, Reasoning was associated with brain activations during NREM sleep and REM sleep after controlling for Verbal and STM factors. As predicted, during NREM sleep, Reasoning factor scores were correlated with the extent of the activation in the bilateral putamen and the paracentral lobule, extending to precuneus. The activation clusters were statistically significant at a threshold of $\mathrm{p}<0.005$ (uncorrected) at the whole-brain level. The paracentral lobule / precuneus cluster survived a more conservative FWE correction $(\mathrm{p}<0.05)$ at the cluster level and the activation of putamen survived SVC correction $(\mathrm{p}<0.05)$ on the right side (SVC for the entire bilateral putamen were defined from the AAL template using WFU Pickatlas (http:// fmri.wfubmc.edu/software/PickAtlas. ). In addition, during REM sleep, we found that the extent of the activation in the midbrain area was associated with Reasoning, (uncorrected $\mathrm{p}<0.005$ at the whole-brain level, and survived FWE correction $(\mathrm{p}<0.05)$ at the cluster level, Table 3$)$.

\section{Correlation between Short-Term Memory Abilities and brain activations modulated by sleep-wake state}

As shown in Fig. 1B, STM factor was associated with brain activation during wake and NREM sleep after controlling 


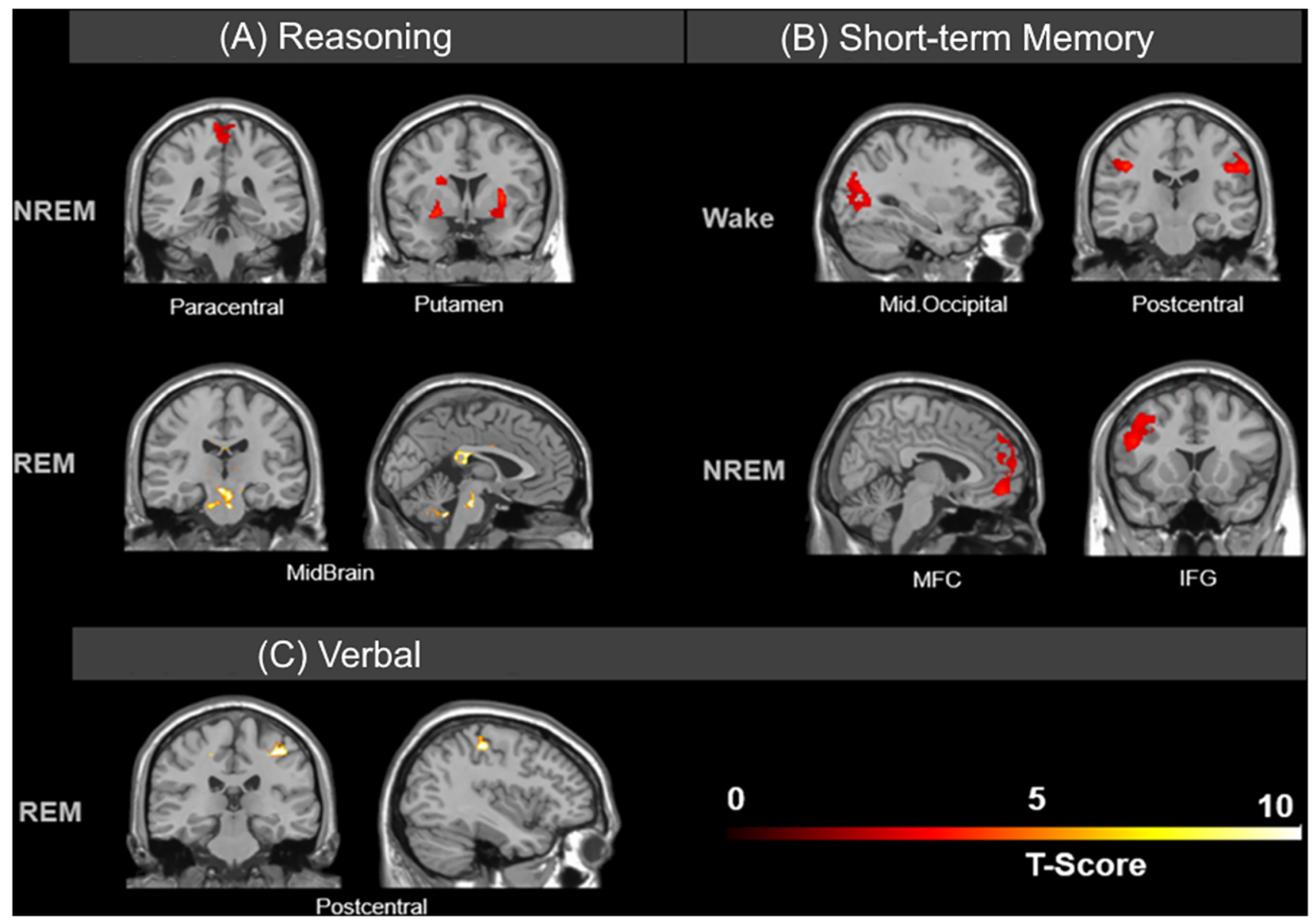

Fig. 1 Whole brain correlation between the extent of brain activations across sleep stages and each cognitive domain. (A) Activation of brain regions correlated with Reasoning during NREM and REM sleep. (B) Activation of brain regions correlated with Short-Term Memory during Wake and NREM sleep. (C) Activation of brain

for Reasoning and Verbal factors. Specifically, during wake, STM was correlated with the extent of the activation of the middle occipital lobe and the bilateral postcentral gyrus. During NREM sleep, STM was correlated with activation in frontal regions, including the medial frontal cortex (MFC) and the inferior frontal gyrus (IFG). These activation clusters were statistically significant at a threshold of $\mathrm{p}<0.005$ (uncorrected) at the whole-brain level, and following more conservative FWE correction $(\mathrm{p}<0.05)$ at the cluster level (Table 3).

\section{Correlation between Verbal Abilities and brain activation modulated by to sleep-wake state}

The Verbal factor was associated with the extent of the activation in the right postcentral gyrus during REM sleep after controlling for Reasoning and STM factors (Fig. 1C, regions correlated with the Verbal factor during REM sleep. Activation clusters were statistically significant at a threshold of uncorrected $\mathrm{p}<0.005$ at the whole-brain level, and FWE correction $\mathrm{p}<0.05$ at the cluster level, except the activation of putamen survived SVC correction $(\mathrm{p}<0.05)$ on the right side during NREM in A

Table 3). The clusters were statistically significant at a threshold of $\mathrm{p}<0.005$ (uncorrected) at the whole-brain level, and following more conservative FWE correction $(\mathrm{p}<0.05)$ at the cluster level.

An additional regression analysis was performed in order to confirm the whole-brain GLM results, and more importantly, in order to assess the effect size of the relationships between cognitive abilities and brain activations across sleep-wake states. We used a post-hoc regions of interest (ROI) analysis, to extract the activation parameter estimates for all significant regions reported (i.e., Fig. 1, Table 3) during the specific stage, and conducted regression analyses for each CBS factor. The standard coefficients, partial correlation coefficients, and p-values are reported in Supplementary Table S2. This follow-up analysis was also performed for the g-factor results. 
Table 3 Whole-brain correlations between CBS scores and brain activations during different sleep-wake states

\begin{tabular}{|c|c|c|c|c|c|}
\hline \multirow[t]{2}{*}{ Brain Regions } & \multicolumn{3}{|c|}{ MNI Coordinates } & \multirow{2}{*}{$\begin{array}{l}\text { Peak } \\
\text { z-score }\end{array}$} & \multirow{2}{*}{$\begin{array}{l}\text { Cluster-level } \\
\text { correction }\end{array}$} \\
\hline & $\mathrm{x}$ & $\mathrm{y}$ & $\mathrm{Z}$ & & \\
\hline
\end{tabular}

\begin{tabular}{|c|c|c|c|c|c|}
\hline \multicolumn{6}{|c|}{ Reasoning \& NREM sleep activation } \\
\hline Paracentral lobule/Pcu & 0 & -34 & 62 & 3.76 & 0.016 \\
\hline R.Putamen & 28 & 4 & 4 & 4.08 & $0.008^{*}$ \\
\hline \multicolumn{6}{|c|}{ Reasoning \& REM sleep activation } \\
\hline Midbrain & 4 & -22 & -22 & 4.36 & 0.003 \\
\hline \multicolumn{6}{|c|}{ Short-Term Memory \& Wake activation } \\
\hline R.postcentral gyrus & 46 & -20 & 32 & 4.05 & 0.05 \\
\hline L.postcentral gyrus & -44 & -28 & 34 & 4.02 & 0.008 \\
\hline Occipital lobe & 32 & -74 & 12 & 3.45 & 0.011 \\
\hline \multicolumn{6}{|c|}{ Short-Term Memory \& NREM activation } \\
\hline MFC & -4 & 52 & -14 & 3.67 & $<0.001$ \\
\hline IFG & -48 & 34 & 10 & 3.81 & $<0.001$ \\
\hline \multicolumn{6}{|c|}{ Verbal \& REM activation } \\
\hline R.postcentral gyrus & 34 & -26 & 48 & 3.95 & 0.034 \\
\hline
\end{tabular}

R. right side; L. left side; MFC: Media Frontal Cortex; IFG: Inferior Frontal gyrus; Pcu: Precuneus; Results significant at $\mathrm{p}<0.05$, Familywise error correction, or following * small volume correction

\section{Correlation between $\mathrm{g}$-factor and brain activations modulated by sleep-wake state}

As shown in Figure S1 and Table S3, during the wakefulness, no positive correlation was found between the brain activations and the CBS g-factor. However, the left posterior cingulate cortex (PCC) was found to be negatively correlated with the CBS g-factor. During NREM sleep, activation of the bilateral lingual gyrus, extending into the left fusiform gyrus was positively correlated with the CBS g-factor. During REM sleep, the midbrain/pons, was positively correlated with the CBS g-factor.

Using a post-hoc regions of interest (ROI) analysis, we extracted the activation parameter estimates for all regions reported (i.e., Figure S1, Table S3) during the specific stages, and conducted regression analyses to confirm the whole brain analyses and to assess the effect size of the relationships between the g-factor scores and brain activations across sleep-wake states (Table S4).

\section{Discussion}

In our current study, Reasoning was associated with brain activation in the paracentral lobule and precuneus as well as bilateral putamen during NREM sleep. Short-Term Memory was associated with activation in the medial frontal cortex and inferior frontal gyrus during NREM sleep, and was also associated with activity in bilateral postcentral gyri, and the occipital lobe during wake. Finally, in a subset of participants who were able to enter REM sleep in the scanner $(\mathrm{N}=8)$, the Reasoning factor was associated with midbrain activation, and the Verbal factor was associated with right postcentral gyrus activation during REM.

\section{Overlap of spindle-locked and non-spindle-locked correlations between BOLD activity and cognitive abilities.}

Our current results show some overlap with our previous spindle-related analysis (Fang, et al., 2019b). In both the previous and current analysis, greater Reasoning was associated with greater activation of the putamen and precuneus in NREM sleep. This finding is also consistent with previous evidence implicating the recruitment of the putamen and precuneus during spindle events (Caporro et al., 2012), and points to their functional significance. Importantly, the associations between Reasoning factor and the thalamus, caudate, cerebellum, and anterior \& middle cingulate, which were observed in the previous spindle-related analysis, did not reach significance in our current analysis. This suggests that the relationship between spindle-evoked brain activity and Reasoning is different from non-spindle-locked brain activity in NREM sleep, as we would expect to see identical patterns of whole brain correlation in both analyses if spindles were not uniquely involved in driving the correlations observed within the aforementioned areas. It has been previously shown that resting state interactions between the default-mode network and the fronto-parietal networks predict individual differences in fluid intelligence (Hearne et al., 2016); our current results suggest that the extent to which components of these networks remain active throughout NREM sleep is related to interindividual differences in Reasoning.

\section{Correlation between the Reasoning factor and brain activations modulated by sleep-wake states}

Greater Reasoning was associated with greater NREM sleep activation in the paracentral lobule, and this association was only observed when BOLD activity was not time-locked to spindles. The paracentral lobule has been associated with decreased activation in autistic individuals compared to healthy controls during Raven's Progressive Matrices, a well-established measure of fluid intelligence (Simard et al., 2015), however, other supporting evidence for this relationship is lacking. The paracentral lobule has been identified as a highly connected node within the structural core of the cerebral cortex (Hagmann et al., 2008), with short, dense tracts forming connections with regions typically associated with the default mode network (with the exclusion of the medial prefrontal cortex). In this light, it is possible that 
correlations between Reasoning factor and activation of the paracentral lobule during NREM sleep may reflect variation in structural connectivity of nodes within fluid intelligencesupporting brain networks. Previous work in awake individuals implicates the frontoparietal network in supporting fluid intelligence (Barbey, 2018). Our current results support the notion that frontal areas are disassociated from cognitionsupporting functional networks during sleep (Muzur et al., 2002).

Participants who experienced REM sleep in the scanner revealed a positive association between Reasoning abilities and the extent of BOLD activation in the midbrain. Although a replication of these results in a larger sample size is warranted, the associations observed in the current study are of utility as they may drive future hypotheses. For example, given its involvement in reinforcement learning, the activation of the midbrain may be associated with the consolidation of previously reinforced strategies or mental models required for reasoning tasks.

\section{Correlation between the Verbal factor and brain activations modulated by sleep-wake states}

A positive association was found between the Verbal factor and BOLD activation in the right postcentral gyrus during REM sleep. The association between the right postcentral gyrus and Verbal ability is unclear, however some studies have found evidence of postcentral activity during REM (Baird et al., 2018; Chow et al., 2013). Nonetheless, these results suggest a functional dissociation between NREM and REM sleep in terms the activation of brain regions supporting distinct cognitive abilities. Interestingly, while the default-mode network has been previously associated with crystalized intelligence during wake (Barbey, 2018), this is not apparent in our current sample.

\section{Correlation between the Short-Term Memory factor and brain activations modulated by sleep-wake states}

The STM factor was associated with activation of the medial-frontal cortex, as well as the inferior frontal gyrus, during NREM sleep. Both of these areas have previously been implicated in short-term memory (Nee \& Jonides, 2014; Nissim et al., 2016), and they have been associated with multiple higher order cognitive domains such as attention and cognitive control (Ridderinkhof et al., 2007; Tops \& Boksem, 2011). In addition, during wake, greater STM abilities were associated with greater BOLD activation in the bilateral postcentral gyri, consistent with recent work showing functional connectivity of the fronto-parietal network associated with trait-like memory abilities (Yamashita et al., 2018). STM was also associated with activation in the middle occipital cortex during wake, possibly as a result of the CBS memory tasks being highly visual in nature. Importantly, no relationships between STM and BOLD activation were observed during the previous spindle-locked analysis (Fang, et al., 2019b), further suggesting a unique relationship between spindle-related networks and Reasoning, wherein networks which support STM seem to show activity during NREM sleep, outside of spindle events.

\section{Correlation between $\mathbf{g}$-factor and brain activations modulated by sleep-wake states}

A component analogous to the traditional ' $\mathrm{g}$ ' factor, representing general cognitive ability across all tasks, showed a negative correlation with the left posterior cingulate cortex during wakefulness. This region, a hub of the default mode network, typically activates during introspective rest / mind wandering, irrespective of the specific contents of consciousness (Leech \& Sharp, 2014). The negative correlation observed here may reflect greater disengagement with the external environment during resting wakefulness in individuals with lower general intelligence. G-factor scores correlated positively with bilateral lingual and left fusiform activation during NREM sleep. These areas support perception and recognition of task-related visual stimuli (Yang et al., 2015), which is a common trait of the 12 tests comprising the g-factor. Finally, the $\mathrm{N}=8$ subjects who achieved REM sleep in the scanner showed a positive correlation between $\mathrm{g}$-factor scores and midbrain activation, similar to the correlation found with the Reasoning factor, though this g-factor correlation also included the pons. It's possible that these correlations represent a cognitive benefit from stronger recruitment of ascending reticular activating areas, which regulate cortical arousal in REM sleep (Fuller et al., 2007). Taken together, these results suggest that general cognitive abilities are related to non-specific functional neuroanatomical correlates across sleep-wake states.

\section{Limitations}

There are a number of limitations with respect to this study. Of the 29 participants who achieved sufficient sleep in the scanner, only 8 experienced REM sleep, limiting the statistical power of our REM analysis. However, this is to our knowledge, the largest sample to-date of participants who have experienced EEG-indexed REM sleep in an MRI scanner. Furthermore, the amount of slowwave sleep was not sufficiently large enough to permit an analysis of stages N2 and N3 sleep separately. Finally, no causal effects can be inferred from our results, as they are entirely correlational. It is still unknown if the relationship between brain activity during sleep stages and intellectual 
ability is a direct effect, or is mediated by other mechanisms. In exploratory studies such as this, it is essential for each finding to be replicated in order to confirm their validity, ideally with a more robust sample size as well as more directed hypothesis testing, using care to limit the possibility of Type I errors during analysis (Elliott et al., 2020). Importantly, these results should be treated with equal interest and caution, as simultaneous EEG-fMRI recordings during deep sleep is extraordinarily difficult to acquire, resulting in the small sample sizes used in our analyses (Turner et al., 2018). Thus, any interpretations made here should be confirmed by replication in future studies. We do hope that the results presented here will serve to inspire future hypotheses made testable in the future with advances in data acquisition and analysis techniques.

\section{Conclusion}

In line with the work by Hampshire et al., (2012) on the relationship between human intelligence and brain activity during wake, and our previous work on the neural correlates of the relationship between sleep spindles and interindividual differences in cognitive abilities (Fang, et al., 2019a, 2019b), the current study supports the hypothesis that the extent of brain activation of dissociable brain areas are related to interindividual differences in distinct, trait-like cognitive abilities (i.e., Reasoning, Short-Term Memory and Verbal). We extend these previous findings to include brain activations across sleep-wake states. Thus, while previous work has shown that there is a robust relationship between spindles and Reasoning (Fang et al., 2017; Fogel et al., 2007; Reynolds et al., 2018; Ujma et al., 2015), the current study suggests that brain activity in other sleep-wake states supports cognitive abilities other than Reasoning (e.g., Verbal and Short-Term Memory) in dissociable brain areas.

Supplementary Information The online version contains supplementary material available at https://doi.org/10.1007/s11682-021-00504-w.

Funding This research was funded by a Canada Excellence Research Chair Grant awarded to author AMO and a Natural Sciences and Engineering Research Council (NSERC) Discovery Grant awarded to author SMF

Availability of data and material Not applicable.

Code availability (software application or custom code) Not applicable.

\section{Declarations}

Conflicts of interest The authors declare that they have no conflict of interest.
Ethics approval This research was approved by the Western University Health Sciences Research Ethics Board.

Consent to participate All participants were made informed and consented to the study procedures.

Consent for publication We consent to the publication of this research.

\section{References}

Allen, P. J., Josephs, O., \& Turner, R. (2000). A method for removing imaging artifact from continuous EEG recorded during functional MRI. NeuroImage, 12(2), 230-239. https://doi.org/10.1006/nimg. 2000.0599

Allen, P. J., Polizzi, G., Krakow, K., Fish, D. R., \& Lemieux, L. (1998). Identification of EEG events in the MR scanner: The problem of pulse artifact and a method for its subtraction. NeuroImage, 8(3), 229-239. https://doi.org/10.1006/nimg.1998.0361

Astill, R. G., Van der Heijden, K. B., Van IJzendoorn, M. H., \& Van Someren, E. J. W. (2012). Sleep cognition and behavioral problems in school-age children: A century of research meta-analyzed. Psychological Bulletin, 138(6), 1109-1138. https://doi.org/10. 1037/a0028204

Baddeley, A. D. (1968). A 3 min reasoning test based on grammatical transformation. Psychonomic Science, 10(10), 341-342.

Baird, B., Castelnovo, A., Riedner, B. A., Lutz, A., Ferrarelli, F., Boly, M., Tononi, G., et al. (2018). Human rapid eye movement sleep shows local increases in low-frequency oscillations and global decreases in high-frequency oscillations compared to resting wakefulness. eNeuro. https://doi.org/10.1523/ENEURO.029318.2018

Barbey, A. K. (2018). Network neuroscience theory of human intelligence. Trends in Cognitive Sciences, 22(1), 8-20. https://doi.org/ 10.1016/j.tics.2017.10.001

Belloc, N. B., \& Breslow, L. (1972). Relationship of physical health status and health practices. Preventive Medicine, 1(3), 409-421. https://doi.org/10.1016/0091-7435(72)90014-X

Bodizs, R., Kis, T., Lazar, A. S., Havran, L., Rigo, P., Clemens, Z., \& Halasz, P. (2005). Prediction of general mental ability based on neural oscillation measures of sleep. Journal of Sleep Research, 14(3), 285-292. https://doi.org/10.1111/j.1365-2869.2005. 00472.x

Bódizs, R., Lázár, A., \& Rigó P. (2008). Correlation of visuospatial memory ability with right parietal EEG spindling during sleep. Acta Physiologica Hungarica, 95(3), 297-306. https://doi.org/10. 1556/APhysiol.95.2008.3.5

Caporro, M., Haneef, Z., Yeh, H. J., Lenartowicz, A., Buttinelli, C., Parvizi, J., \& Stern, J. M. (2012). Functional MRI of sleep spindles and K-complexes. Clinical Neurophysiology, 123(2), 303309. https://doi.org/10.1016/j.clinph.2011.06.018

Cattell, R. B. (1940). A culture-free intelligence test. I. Journal of Educational Psychology, 31(3), 161-179. https://doi.org/10.1037/ h0059043

Chow, H. M., Horovitz, S. G., Carr, W. S., Picchioni, D., Coddington, N., Fukunaga, M., et al. (2013). Rhythmic alternating patterns of brain activity distinguish rapid eye movement sleep from other states of consciousness. Proceedings of the National Academy of Sciences of the United States of America, 110(25), 10300-10305. https://doi.org/10.1073/pnas.1217691110

Collins, P., Roberts, A. C., Dias, R., Everitt, B. J., \& Robbins, T. W. (1998). Perseveration and strategy in a novel spatial self-ordered sequencing task for nonhuman primates: Effects of excitotoxic 
lesions and dopamine depletions of the prefrontal cortex. Journal of Cognitive Neuroscience, 10(3), 332-354. https://doi.org/ $10.1162 / 089892998562771$

Corsi, P. M. (1972). Human memory and the medial temporal region of the brain.

Della Monica, C., Johnsen, S., Atzori, G., Groeger, J. A., \& Dijk, D.-J. (2018). Rapid eye movement sleep, sleep continuity and slow wave sleep as predictors of cognition, mood, and subjective sleep quality in healthy men and women, aged $20-84$ years. Frontiers in Psychiatry, 9, 255. https://doi.org/10.3389/fpsyt.2018.00255

Delorme, A., \& Makeig, S. (2004). EEGLAB: An open source toolbox for analysis of single-trial EEG dynamics including independent component analysis. Journal of Neuroscience Methods, 134(1), 9-21. https://doi.org/10.1016/j.jneumeth.2003.10.009

Diekelmann, S. (2014). Sleep for cognitive enhancement. Frontiers in Systems Neuroscience, 8, 46. https://doi.org/10.3389/fnsys.2014. 00046

Dzierzewski, J. M., Dautovich, N., \& Ravyts, S. (2018). Sleep and cognition in older adults. Sleep Medicine Clinics, 13(1), 93-106. https://doi.org/10.1016/j.jsmc.2017.09.009

Elliott, M. L., Knodt, A. R., Ireland, D., Morris, M. L., Poulton, R., Ramrakha, S., et al. (2020). What Is the test-retest reliability of common task-functional MRI measures? new empirical evidence and a meta-analysis. Psychological Science, 31(7), 792-806. https://doi.org/10.1177/0956797620916786

Fang, Z., Ray, L. B., Houldin, E., Smith, D., Owen, A. M., \& Fogel, S. M. (2019a). Sleep Spindle-dependent functional connectivity correlates with cognitive abilities. Journal of Cognitive Neuroscience. https://doi.org/10.1162/jocn_a_01488

Fang, Z., Ray, L. B., Owen, A. M., \& Fogel, S. M. (2019b). Brain activation time-locked to sleep spindles associated with human cognitive abilities. Frontiers in Neuroscience, 13, 46. https://doi. org/10.3389/fnins.2019.00046

Fang, Z., Sergeeva, V., Ray, L. B., Viczko, J., Owen, A. M., \& Fogel, S. M. (2017). Sleep spindles and intellectual ability: Epiphenomenon or directly related? Journal of Cognitive Neuroscience, 29(1), 167-182. https://doi.org/10.1162/jocn_a_01034

Fogel, S. M., Nader, R., Cote, K. A., \& Smith, C. T. (2007). Sleep spindles and learning potential. Behavioral Neuroscience, 121(1), 1-10. https://doi.org/10.1037/0735-7044.121.1.1

Fogel, S. M., \& Smith, C. T. (2006). Learning-dependent changes in sleep spindles and Stage 2 sleep. Journal of Sleep Research, 15(3), 250-255. https://doi.org/10.1111/j.1365-2869.2006.00522.x

Folstein, M. F., Folstein, S. E., \& McHugh, P. R. (1975). Mini-mental state". A practical method for grading the cognitive state of patients for the clinician. Journal of Psychiatric Research, 12(3), 189-198. https://doi.org/10.1016/0022-3956(75)90026-6

Friston, K. J., Williams, S., Howard, R., Frackowiak, R. S., \& Turner, R. (1996). Movement-related effects in fMRI time-series. Magnetic Resonance in Medicine, 35(3), 346-355. https://doi.org/10. 1002/mrm.1910350312

Fuller, P. M., Saper, C. B., \& Lu, J. (2007). The pontine REM switch: Past and present. The Journal of Physiology, 584(Pt 3), 735-741. https://doi.org/10.1113/jphysiol.2007.140160

Gould, R. L., Brown, R. G., Owen, A. M., Bullmore, E. T., \& Howard, R. J. (2006). Task-induced deactivations during successful paired associates learning: An effect of age but not Alzheimer's disease. NeuroImage, 31(2), 818-831. https://doi.org/10.1016/j. neuroimage.2005.12.045

Hagmann, P., Cammoun, L., Gigandet, X., Meuli, R., Honey, C. J., Wedeen, V. J., \& Sporns, O. (2008). Mapping the structural core of human cerebral cortex. PLoS Biology, 6(7), e159. https://doi. org/10.1371/journal.pbio.0060159

Hampshire, A., Highfield, R. R., Parkin, B. L., \& Owen, A. M. (2012). Fractionating human intelligence. Neuron, 76(6), 1225-1237. https://doi.org/10.1016/j.neuron.2012.06.022
Hearne, L. J., Mattingley, J. B., \& Cocchi, L. (2016). Functional brain networks related to individual differences in human intelligence at rest. Scientific Reports, 6, 32328. https://doi.org/10.1038/srep3 2328

Iber, C., Ancoli-Israel, S., Chesson, A. L., \& Quan, S. F. (2007). The AASM manual for the scoring of sleep and associated events: rules, terminology and technical specifications (1st ed., p. 59). American Academy of Sleep Medicine, Westchester, IL.

IBM Corp. Released 2019. IBM SPSS Statistics for Windows, Version 26.0. Armonk, NY: IBM Corp

Inoue, K., Kawashima, R., Satoh, K., Kinomura, S., Sugiura, M., Goto, R., et al. (2000). A PET study of visuomotor learning under optical rotation. NeuroImage, 11(5 Pt 1), 505-516. https://doi.org/10.1006/nimg.2000.0554

Krause, A. J., Simon, E. B., Mander, B. A., Greer, S. M., Saletin, J. M., Goldstein-Piekarski, A. N., \& Walker, M. P. (2017). The sleep-deprived human brain. Nature Reviews Neuroscience, 18(7), 404-418.

Leech, R., \& Sharp, D. J. (2014). The role of the posterior cingulate cortex in cognition and disease. Brain A Journal of Neurology, 137(Pt 1), 12-32. https://doi.org/10.1093/brain/awt162

Lim, J., \& Dinges, D. F. (2010). A meta-analysis of the impact of short-term sleep deprivation on cognitive variables. Psychological Bulletin, 136(3), 375-389. https://doi.org/10.1037/a0018883

Lo, J. C., Groeger, J. A., Cheng, G. H., Dijk, D-J., \& Chee, M. W. L. (2016) Self-reported sleep duration and cognitive performance in older adults: a systematic review and meta-analysis. Sleep Medicine, 1787-1798. https://doi.org/10.1016/j.sleep.2015.08.021

Mantini, D., Perrucci, M. G., Cugini, S., Ferretti, A., Romani, G. L., \& Del Gratta, C. (2007). Complete artifact removal for EEG recorded during continuous fMRI using independent component analysis. NeuroImage, 34(2), 598-607. https://doi.org/10.1016/j. neuroimage.2006.09.037

Muzur, A., Pace-Schott, E. F., \& Hobson, J. A. (2002). The prefrontal cortex in sleep. Trends in Cognitive Sciences, 6(11), 475-481. https://doi.org/10.1016/s1364-6613(02)01992-7

Nee, D. E., \& Jonides, J. (2014). Frontal-medial temporal interactions mediate transitions among representational states in short-term memory. The Journal of Neuroscience, 34(23), 7964-7975. https://doi.org/10.1523/JNEUROSCI.0130-14.2014

Nissim, N. R., O’Shea, A. M., Bryant, V., Porges, E. C., Cohen, R., \& Woods, A. J. (2016). Frontal structural neural correlates of working memory performance in older adults. Frontiers in Aging Neuroscience, 8, 328. https://doi.org/10.3389/fnagi.2016.00328

Pesonen, A-K., Ujma, P., Halonen, R. Räikkönen, K., \& Kuula, L. (2019). The associations between spindle characteristics and cognitive ability in a large adolescent birth cohort. Intelligence, 7213-7219. https://doi.org/10.1016/j.intell.2018.11.004

Reynolds, C. M., Short, M. A., \& Gradisar, M. (2018). Sleep spindles and cognitive performance across adolescence: A meta-analytic review. Journal of Adolescence, 66, 55-70. https://doi.org/10. 1016/j.adolescence.2018.04.003

Ridderinkhof, K. R., Nieuwenhuis, S., \& Braver, T. S. (2007). Medial frontal cortex function: An introduction and overview. Cognitive, Affective \& Behavioral Neuroscience, 7(4), 261-265. https://doi. org/10.3758/cabn.7.4.261

Santarnecchi, E., Emmendorfer, A., Tadayon, S., Rossi, S., Rossi, A., \& Pascual-Leone, A. (2017). Network connectivity correlates of variability in fluid intelligence performance. Intelligence, 65 , 35-47. https://doi.org/10.1016/j.intell.2017.10.002

Schabus, M., Gruber, G., Parapatics, S., Cornelia, S., Sauter, C., Klösch, G., Anderer, P., Klimesch, W., Saletu, B., \& Zeitlhofer, J. (2004). Sleep spindles and their significance for declarative memory consolidation. Sleep, 27(8), 1479-1485. https://doi.org/ 10.1093/sleep/27.7.1479 
Schabus, M., Hödlmoser, K., Gruber, G., Sauter, C., Anderer, P., Klösch, G., Parapatics, S., Saletu, B., Klimesch, W., \& Zeitlhofer, J. (2006). Sleep spindle-related activity in the human EEG and its relation to general cognitive and learning abilities. European Journal of Neuroscience, 23(7), 1738-1746. https://doi.org/10. 1111/j.1460-9568.2006.04694.x

Shallice, T. (1982). Specific impairments of planning. Philosophical Transactions of the Royal Society of London Series B, Biological Sciences, 298(1089), 199-209. https://doi.org/10.1098/rstb. 1982.0082

Silverman, I., Choi, J., \& Mackewn, A. (2000). Evolved mechanisms underlying wayfinding: Further studies on the hunter-gatherer theory of spatial sex differences. Evolution and Human Behavior, 21(3), 201-213.

Simard, I., Luck, D., Mottron, L., Zeffiro, T. A., \& Soulières, I. (2015). Autistic fluid intelligence: Increased reliance on visual functional connectivity with diminished modulation of coupling by task difficulty. NeuroImage Clinical, 9, 467-478. https://doi.org/10.1016/j. nicl.2015.09.007

Smith, C. T., Nixon, M. R., \& Nader, R. S. (2004). Posttraining increases in REM sleep intensity implicate REM sleep in memory processing and provide a biological marker of learning potential. Learning \& Memory, 11(6), 714-719. https://doi.org/10.1101/lm. 74904

Srivastava, G., Crottaz-Herbette, S., Lau, K. M., Glover, G. H., \& Menon, V. (2005). ICA-based procedures for removing ballistocardiogram artifacts from EEG data acquired in the MRI scanner. NeuroImage, 24(1), 50-60. https://doi.org/10.1016/j.neuroimage. 2004.09.041

Stevens, A. A., Tappon, S. C., Garg, A., \& Fair, D. A. (2012). Functional brain network modularity captures inter- and intra-individual variation in working memory capacity. PLOS ONE, 7(1), e30468. https://doi.org/10.1371/journal.pone.0030468

Stroop, J. R. (1935). Studies of interference in serial verbal reactions. Journal of Experimental Psychology, 18(6), 643-662. https://doi.org/10.1037/h0054651

Tarokh, L., Saletin, J. M., \& Carskadon, M. A. (2016). Sleep in adolescence: Physiology cognition and mental health. Neuroscience \& Biobehavioral Reviews, 70182-70188. https://doi.org/10.1016/j. neubiorev.2016.08.008

Tops, M., \& Boksem, M. A. S. (2011). A potential role of the inferior frontal gyrus and anterior insula in cognitive control, brain rhythms, and event-related potentials. Frontiers in Psychology, 2, 330. https://doi.org/10.3389/fpsyg.2011.00330

Treisman, A. M., \& Gelade, G. (1980). A feature-integration theory of attention. Cognitive Psychology, 12(1), 97-136. https://doi.org/ 10.1016/0010-0285(80)90005-5

Turner, B. O., Paul, E. J., Miller, M. B., \& Barbey, A. K. (2018). Small sample sizes reduce the replicability of task-based fMRI studies. Communications Biology, 1, 62. https://doi.org/10.1038/ s42003-018-0073-z

Ujma, P. P. (2018) Sleep spindles and general cognitive ability - A meta-analysis. Sleep Spindles \& Cortical Up States, 1-17. https:// doi.org/10.1556/2053.2.2018.01

Ujma, P., Konrad, B.N., Lisa, Genzel, L., Bleifuss, A., Simor, P., Pótári, A., Körmendi, J., Gombos, F., Steiger, A., Bódizs, R., \& Dresler, M. (2014). Sleep spindles and intelligence: Evidence for a sexual dimorphism. The Journal of Neuroscience, 34(49), 16358-16368. https://doi.org/10.1523/JNEUROSCI.1857-14.2014

Ujma, P. P., Bódizs, R., \& Dresler, M. (2020). Sleep and intelligence: critical review and future directions. Current Opinion in Behavioral Sciences, 33109-33117. https://doi.org/10.1016/j.cobeha. 2020.01.009

Ujma, P. P., Bódizs, R., Gombos, F., Stintzing, J., Konrad, B. N., Genzel, L., et al. (2015). Nap sleep spindle correlates of intelligence. Scientific Reports, 5, 17159. https://doi.org/10.1038/srep17159

Ujma, P. P., Konrad, B. N., Gombos, F., Simor, P., Pótári, A., Genzel, L., et al. (2017). The sleep EEG spectrum is a sexually dimorphic marker of general intelligence. Scientific Reports, 7(1), 18070. https://doi.org/10.1038/s41598-017-18124-0

Walker, M. P. (2009) The role of slow wave sleep in memory processing. Journal of Clinical Sleep Medicine, 5 (2 suppl). https://doi. org/10.5664/jcsm.5.2S.S20

Wecshler, D. (1981). WAIS-R manual: Wechsler adult intelligence scale-revised. Psychological Corporation.

Wild, C. J., Nichols, E. S., Battista, M. E., Stojanoski, B., \& Owen, A. M. (2018). Dissociable effects of self-reported daily sleep duration on high-level cognitive abilities. Sleep. https://doi.org/10.1093/ sleep/zsy 182

Worley, S. L. (2018). The extraordinary importance of sleep: The detrimental effects of inadequate sleep on health and public safety drive an explosion of sleep research. $P \& T:$ A Peer-Reviewed Journal for Formulary Management, 43(12), 758-763.

Yamashita, M., Yoshihara, Y., Hashimoto, R., Yahata, N., Ichikawa, N., Sakai, Y., et al. (2018). A prediction model of working memory across health and psychiatric disease using whole-brain functional connectivity. eLife. https://doi.org/10.7554/eLife.38844

Yang, Y.-L., Deng, H.-X., Xing, G.-Y., Xia, X.-L., \& Li, H.-F. (2015). Brain functional network connectivity based on a visual task: Visual information processing-related brain regions are significantly activated in the task state. Neural Regeneration Research, 10(2), 298-307. https://doi.org/10.4103/1673-5374.152386

Publisher's Note Springer Nature remains neutral with regard to jurisdictional claims in published maps and institutional affiliations. 